\title{
Platelet to lymphocyte ratio as an independent prognostic indicator for prostate cancer patients receiving androgen deprivation therapy
}

Yanqing Wang ${ }^{1+}$, Fan Xu ${ }^{1+}$, Jiahua Pan ${ }^{1}$, Yinjie Zhu' ${ }^{1}$ Xiaoguang Shao ${ }^{1}$, Jianjun Sha', Zezhou Wang ${ }^{2}$, Yong Cai ${ }^{2}$, Qiang Liu ${ }^{3}$, Baijun Dong ${ }^{1 *}$, Wei Xue ${ }^{1 *}$ and Yiran Huang ${ }^{1}$

\begin{abstract}
Background: Platelet to Lymphocyte ratio $(P L R)$ is thought to be associated with a worse outcome in multiple types of cancer. However, the prognostic significance of PLR has not been investigated in the prostate cancer (PCa) patients receiving hormonal therapy. The objective of this study was to determine the prognostic value of PLR in PCa patients treated with androgen deprivation therapy (ADT).

Methods: Two-hundred-ninety prostate cancer patients who had undergone ADT as first-line therapy were retrospectively analyzed. The blood cell counts were performed at the time of diagnosis. PLR was calculated as the ratio of platelet count to lymphocyte count. Patients were categorized in two groups using a cut-off point of 117.58 as calculated by the receiver-operating curve analysis. Correlations between PLR and clinical characteristics were analyzed. Meanwhile, univariate and multivariate cox regression analyses were performed to determine the associations of PLR with progression-free survival (PFS), cancer-specific survival (CSS) and overall survival (OS). Prognostic accuracy was evaluated with the Harrell concordance index.

Results: The differences of age, serum prostate-specific antigen (PSA) level, Gleason score, risk stratification and incidence of metastasis between low PLR group $(<117.58)$ and high PLR group $(\geq 117.58)$ were not statistically significant $(p>0.05)$. Multivariate analyses identified PLR as an independent prognostic factor for PFS (hazard ratio $(H R)=1.581, p=0.013)$, CSS (HR $=1.768, p=0.037)$ and OS $(H R=1.650, p=0.044)$. The addition of PLR to the final model improved predictive accuracy (c-index: $0.747,0.801$ and 0.768 ) for PFS, CSS and OS compared with the clinicopathological base model (c-index: 0.730, 0.778 and 0.746), which included Gleason score and incidence of metastasis.

Conclusions: PLR might play a significant role in the prognosis of PCa patients treated with ADT. Thus, we recommend adding PLR to traditional prognostic model to improve the predictive accuracy.
\end{abstract}

Keywords: Prostate cancer, PLR, Prognostic factor, Survival

\footnotetext{
* Correspondence: dongbaijun@hotmail.com; uroxuewei@163.com

${ }^{\dagger}$ Equal contributors

'Department of Urology, Renji Hospital, School of Medicine, Shanghai Jiao

Tong University, 1630 Dongfang Road, Pudong District, Shanghai 200127,

People's Republic of China

Full list of author information is available at the end of the article
}

\section{) Bïomed Central}

(c) 2016 Wang et al. Open Access This article is distributed under the terms of the Creative Commons Attribution 4.0 International License (http://creativecommons.org/licenses/by/4.0/), which permits unrestricted use, distribution, and reproduction in any medium, provided you give appropriate credit to the original author(s) and the source, provide a link to the Creative Commons license, and indicate if changes were made. The Creative Commons Public Domain Dedication waiver (http://creativecommons.org/publicdomain/zero/1.0/) applies to the data made available in this article, unless otherwise stated. 


\section{Background}

Prostate cancer (PCa) is the most commonly diagnosed cancer and the second leading cause of cancer death in men in the United States [1]. Androgen deprivation therapy (ADT) is the mainstay of therapy for patients with locally advanced or metastatic PCa or patients with early-stage disease who are ineligible for local regional treatments due to health disparity [2].

A growing body of evidence suggests that inflammation might have a major role in the tumorigenesis and progression of $\mathrm{PCa}$ [3-5]. Low serum neutrophil count predicts a positive prostate biopsy [6]. The neutrophil to lymphocyte ratio (NLR) seems to represent an independent prognostic marker in patients with $\mathrm{PCa}$ [7]. Similarly, platelet to lymphocyte ratio (PLR) is also a systemic inflammation-based parameter. Numerous studies have revealed that high pretreatment PLR independently predicts poor prognosis in patients with tumors including gastric cancer [8], pancreatic cancer [9], ovarian cancer [10], colorectal cancer [11], non-small cell lung cancer [12], hepatocellular carcinoma [13], renal cell cancer [14], esophageal cancer [15]. Yuksel $\mathrm{OH}$ et al. [16] and Kaynar M et al. [17] reported that PLR could be used to distinguish benign prostatic hyperplasia and prostate cancer, in support of its diagnostic value. Langsenlehner $\mathrm{T}$ et al. [18] showed a significant association between PLR and prognosis of PCa patients who underwent radiation therapy. However, whether PLR plays an important role in the prognosis of $\mathrm{PCa}$ treated with ADT has not been reported.

Platelet and lymphocyte counts are routinely performed in most clinical laboratories worldwide, therefore we evaluated whether pretreatment PLR could predict the clinical outcome of $\mathrm{PCa}$ patients treated with ADT.

\section{Methods}

\section{Study population}

After obtaining approval from the Ethics Committee at the Renji Hospital, Shanghai Jiao Tong University School of Medicine, and informed consent from patients, medical records of 325 prostate cancer patients who had undergone $\mathrm{ADT}$ as first-line therapy at the Renji Hospital between January 2010 and December 2014 were retrospectively reviewed. We excluded patients with coagulation-related diseases, inflammatory diseases, autoimmune diseases, cerebrovascular diseases, other types of cancer, and those patients lost to follow-up. We finally assembled a cohort of 290 prostate cancer patients who had blood cell counts performed within 2 weeks before prostate biopsy.

\section{Clinical and pathological evaluation}

Medical data on clinical characteristics including age, serum prostate-specific antigen (PSA) level and blood cell counts at diagnosis, clinical tumor stage, biopsy Gleason score and follow-up information were collected. The pathologic slides were re-reviewed by the urologic pathologists, and the Gleason scores were obtained from the original pathology reports. For clinical tumor stage, patients underwent pelvic Computed Tomography (CT) or Magnetic Resonance Imaging (MRI). Radionuclide bone scan was performed to determine whether there was bone metastasis. PCa patients were stratified into low-, intermediate-, and high-risk groups according to the EAU guidelines [19]: low-risk group, PSA $<10 \mu \mathrm{g} / \mathrm{L}$ and Gleason Score $<7$ and cT1c-2a; intermediate-risk group, PSA $10-20 \mu \mathrm{g} / \mathrm{L}$ or Gleason Score 7 or cT2b-2c; and high-risk group, PSA $>20 \mu \mathrm{g} / \mathrm{L}$ or Gleason Score 810 or $>$ cT2c.

Eligible patients were treated with continuous ADT as first-line therapy, including castration and antiandrogen therapy. Castration involved surgical or medical castration by using a luteinizing hormone releasing hormone (LHRH) agonist, such as goserelin $3.6 \mathrm{mg}$, administered subcutaneously every month. Antiandrogen therapy was by bicalutamide tablets $50 \mathrm{mg}$ per day orally or flutamide 3 times a day, $250 \mathrm{mg}$ each time orally.

\section{Follow-up}

Patients were followed for survival information every 3 months. Duration of the follow-up was assessed from the date of treatment until the last follow-up (June 2015) or death, which was defined as cancer-related or a different cause. Progression was defined as castrationresistance or death, and the castration-resistance was judged according to the EAU guidelines [20]. The median follow-up duration was 37.0 months (IQR, 24.050.3).

\section{Laboratory assays}

Venous blood samples were collected before the prostate biopsy. Pre-biopsy platelet and lymphocyte counts were performed as part of routine clinical procedures to exclude coagulation disorders or presence of acute infection.

\section{Statistical analysis}

The Wilcoxon Signed Rank test was used to interrogate the median with interquartile ranges (IQRs) between PLR and clinical characteristics, while chi-squared tests were used for categorical variables. The cut-off value for the continuous variable PLR was determined by applying a receiver operating characteristics curve analysis to test all possible cut-offs that would separate between patients' survival and cancer-related death. The survival distributions, including progression-free survival (PFS), cancer-specific survival (CSS) and overall survival (OS) were estimated by the Kaplan-Meier method and 
Table 1 Clinical characteristics of prostate cancer patients treated with ADT $(n=290)$

\begin{tabular}{lc}
\hline Parameters & No. of patients (\%) \\
\hline Age (median, interquartile range), years & $75(67-79)$ \\
PSA (median, interquartile range), $\mu \mathrm{g} / \mathrm{L}$ & $100.00(31.13-198.50)$ \\
Gleason Score & \\
$\quad<7$ & $36(12.41)$ \\
7 & $108(37.24)$ \\
$>7$ & $146(50.35)$ \\
Metastasis & \\
$\quad$ No & $159(54.83)$ \\
$\quad$ Yes & $131(45.17)$ \\
Risk Stratification & \\
Low & $1(0.34)$ \\
Intermediate & $27(9.31)$ \\
High & $262(90.35)$ \\
Platelet (median, interquartile range), 109/L & $181.50(145.00-215.25)$ \\
Lymphocyte (median, interquartile range), $109 / \mathrm{L}$ & $1.54(1.21-1.89)$ \\
PLR & $117.46(87.26-154.57)$ \\
Progression-free survival & $126(43.45)$ \\
Cancer-specific survival & $60(20.69)$ \\
Overall survival & $70(24.14)$ \\
Follow-up time (months) & $37.00(24.00-50.30)$ \\
\hline Abbrevations: PSA prosta
\end{tabular}

Abbreviations: PSA prostate-specific antigen

compared by a log-rank test, and subgroup analyses were taken according to Gleason score and incidence of metastasis. PFS was calculated from the date of prostate biopsy to the date of disease progression or the time of the last follow-up. The effect of PLR on PFS, CSS and OS were examined using cox proportional hazard regression models. All variables including PLR with a $p$ value $<0.05$ on univariate analyses were entered into multivariate stepwise cox regression analyses. Hazard ratio $(\mathrm{HR})$ and $95 \%$ confidence interval $(\mathrm{CI})$ were computed. To examine whether PLR can provide additional prognostic power when combined with basic clinical variables, we built predictive model and calculated the c- index by integrating clinical variables with PLR using the $\mathrm{R}$ package "survival." For each core set, we randomly extracted $20 \%$ samples as the test set to generate a cindex, and the above procedure was repeated 100 times to generate $100 \mathrm{c}$-indexes. Then, we used the Wilcoxon signed rank test to calculate the $p$ value. All tests were two-sided. Differences were considered to be statistically significant if $p<0.05$. Statistical analysis was carried out using SPSS, version 19.0.

\section{Results}

\section{Clinical characteristics}

The clinical characteristics of the patients are detailed in Table 1 . The median age of the patients was 75 years old (IQR, 67-79).

\section{Association between PLR and clinical and pathological characteristics}

Based on ROC curve for survival analysis (CSS), the best cut-off value for PLR was 117.58, and all patients were divided into either low PLR group ( $n=146,50.34 \%)$ or high PLR group ( $n=144,49.66 \%)$. The differences of age, serum PSA level, Gleason score, risk stratification and incidence of metastasis between low PLR group and high PLR group were not significant $(p>0.05)$. (Table 2).

\section{Association between PLR and prognosis of PCa}

The median follow-up duration was 37.0 months, out of 290 patients with usable follow-up information, 126 (43.45\%) patients experienced disease progression, and $70(24.14 \%)$ patients died, including 60 (20.69 \%) patients died of $\mathrm{PCa}$ at the end of the last follow-up.

The patients with high PLR had a significantly worse survival than those with low PLR with regard to PFS, CSS and OS (Log-rank test, each $P<0.05$, Fig. 1). As shown in Figs. 2 and 3, in the subgroup of patients with Gleason score $>7$ or bone metastasis, high PLR group predicted the worse PFS, CSS and OS (Log-rank test, each $P<0.01)$. However in the subgroup of Gleason score $\leq 7$ or non-metastasis, the prognostic differences of clinical outcome were not significant between high PLR

Table 2 Clinical characteristics of prostate cancer patients according to PLR

\begin{tabular}{|c|c|c|c|}
\hline \multirow[t]{2}{*}{ Parameters } & \multicolumn{2}{|l|}{ PLR } & \multirow[t]{2}{*}{$P$-value } \\
\hline & $<117.58(n=14650.34 \%)$ & $\geq 117.58(n=14449.66 \%)$ & \\
\hline Age (median, interquartile range), years & $76(66-79)$ & $75(68-80)$ & 0.632 \\
\hline PSA (median, interquartile range), $\mu \mathrm{g} / \mathrm{L}$ & $100.00(28.30-170.00)$ & $100.00(33.05-213.00)$ & 0.625 \\
\hline Gleason Score $(\leq 7 />7)$ & $76 / 70$ & $68 / 76$ & 0.410 \\
\hline Metastasis (no/yes) & $87 / 59$ & $72 / 72$ & 0.100 \\
\hline Risk Stratification (low intermediate/high) & $15 / 131$ & 13/131 & 0.719 \\
\hline Platelet (median, interquartile range), 109/L & $159.50(134.00-198.00)$ & $201.00(167.50-238.50)$ & $<0.001$ \\
\hline Lymphocyte (median, interquartile range), 109/L & $1.84(1.57-2.21)$ & $1.24(1.02-1.51)$ & $<0.001$ \\
\hline
\end{tabular}

Abbreviations: PSA, prostate-specific antigen 

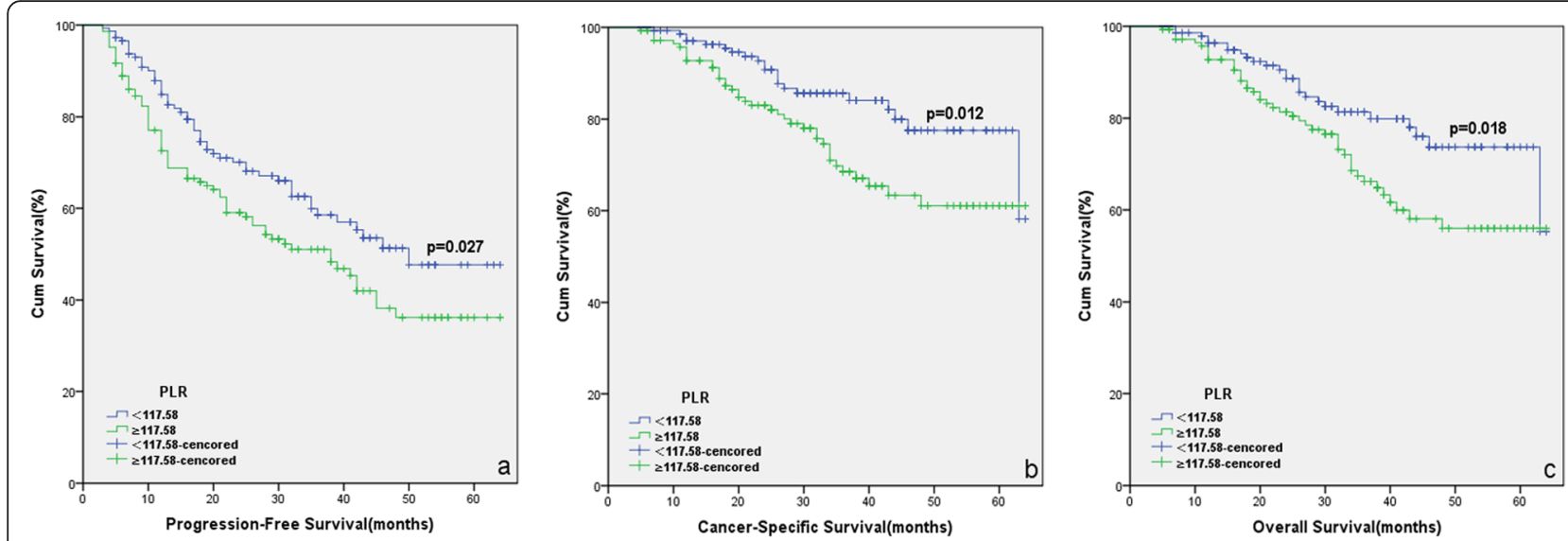

Fig. 1 Kaplan-Meier curves for survival of prostate cancer patients according to PLR. a Progression-free survival (PFS), b Cancer-specific survival (CSS) and c Overall survival (OS)

group and low PLR group (Log-rank test, each $P>0.05$ ). Univariate and multivariate cox regression analyses (stepwise analysis) of the factors influencing PFS, CSS and OS were presented in Tables 3 and 4. Univariate analyses demonstrated that serum PSA level, Gleason score, incidence of metastasis, PLR were significant predictors for PFS, CSS and OS (each $P<0.05$ ), but age and risk stratification were significant predictors for PFS, not for CSS and OS. In the multivariate analyses, we identified that age, Gleason score, incidence of metastasis and PLR were independent prognostic factors for PFS, while Gleason score, incidence of metastasis and
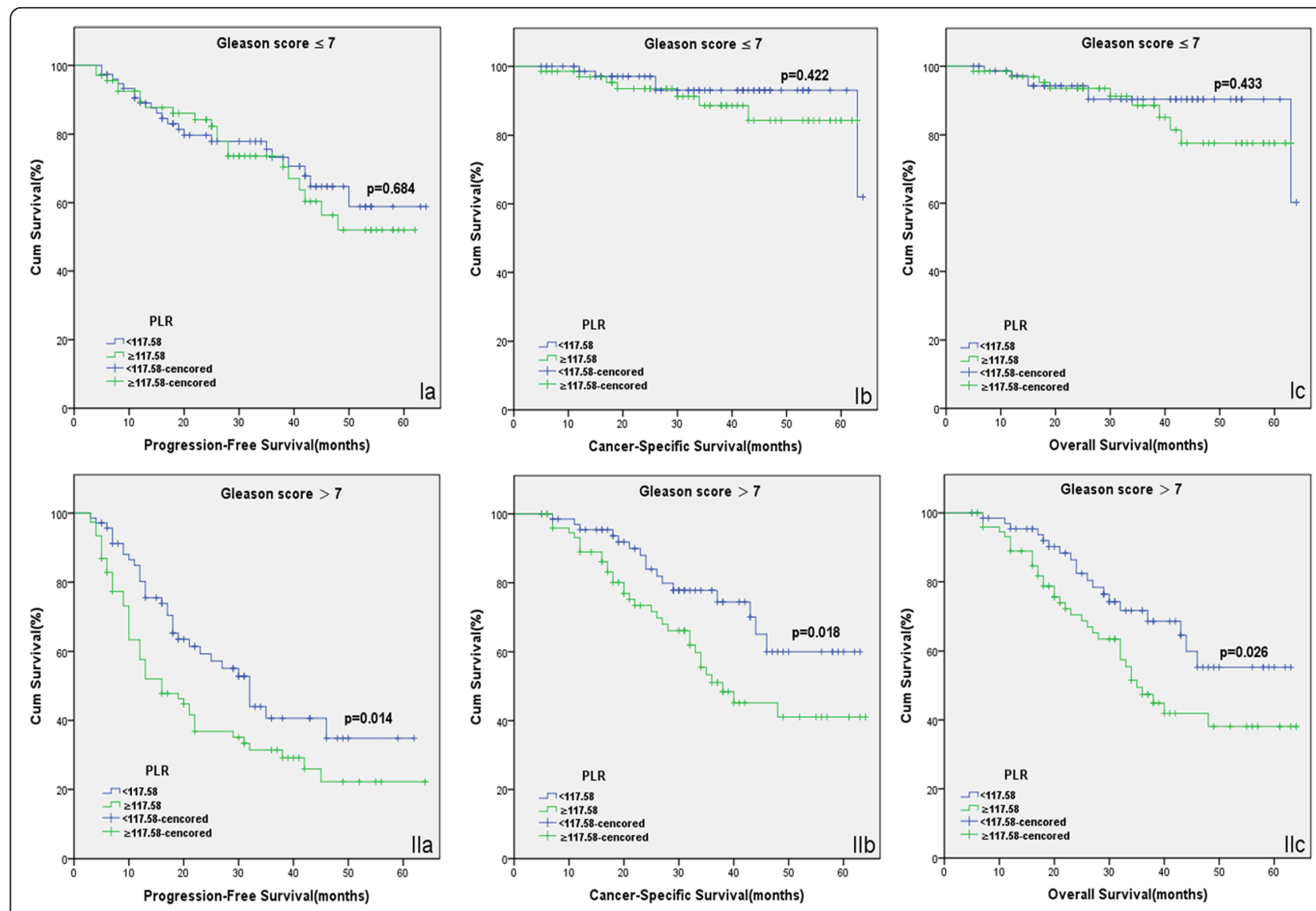

Fig. 2 Kaplan-Meier survival curves stratified by PLR in prostate cancer patients with Gleason score $\leq 7$ (I) and Gleason score $>7$ (II). a Progressionfree survival (PFS), b Cancer-specific survival (CSS) and c Overall survival (OS) 


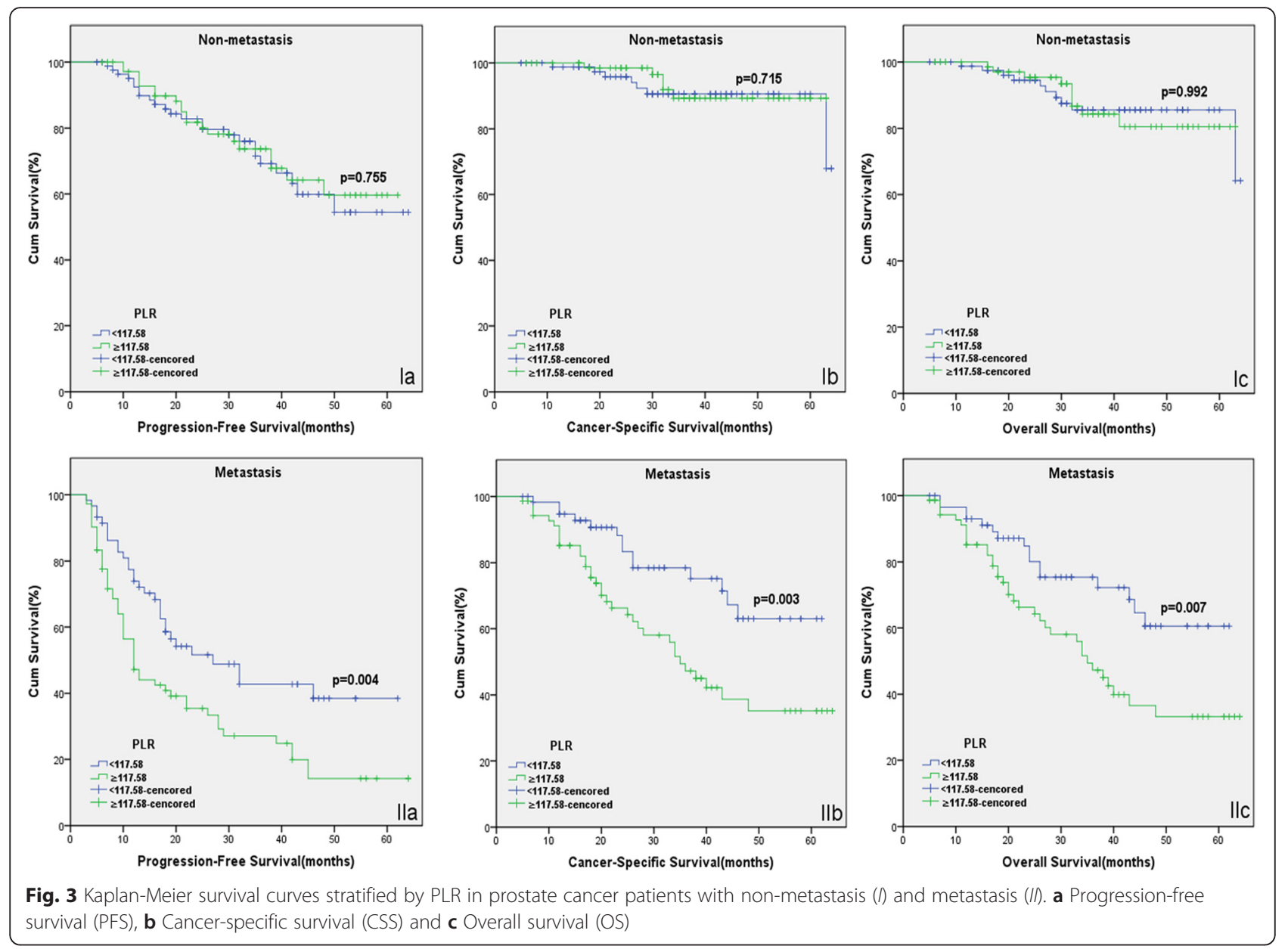

PLR were independent prognostic factors for CSS and OS. The HRs of PLR were 1.581 (95 \% CI 1.100-2.272) for PFS, 1.768 (95 \% CI 1.036-3.015) for CSS and 1.650 (95 \% CI 1.013-2.687) for OS, respectively.

The predictive accuracy was calculated with and without the inclusion of PLR. With the base model, including the traditional predictor variables of Gleason score and incidence of metastasis, predictive accuracy for PFS, CSS and OS was $73.0 \%$ (IQR, 71.1-77.1 \%), $77.8 \%$ (IQR, $73.1-81.9 \%$ ) and $74.6 \%$ (IQR, 71.8-77.7 \%). Our new integrated model with the addition of PLR, predictive accuracy for PFS, CSS and OS was $74.7 \%$ (IQR, 72.3$77.8 \%$ ), $80.1 \%$ (IQR, 76.7-84.2 \%) and $76.8 \%$ (IQR, 73.7-79.9\%), respectively. Notably, our integrated model showed statistically significantly improved predictive power compared to the base model (each $p<0.001$ ).

\section{Discussion}

Despite recent progress in the identification of genetic and molecular alterations in prostate cancer $(\mathrm{PCa})$, the routine prognostic risk assessment of $\mathrm{PCa}$ patients currently relies on traditional clinicopathological prognostic factors, including Gleason score, clinical tumor stage, and serum PSA level at the time of diagnosis, which are used for stratify the patients into the low-, intermediate-, or high-risk group [19]. The predictive accuracy of this traditional prognostic model need be further improved by the incorporation of novel prognostic biomarkers.

The platelet and lymphocyte counts are routinely measured blood-based parameters. In this large cohort of PCa patients treated with androgen deprivation therapy (ADT), we found that pretreatment high platelet to lymphocyte ratio (PLR) was associated with increased risk for disease recurrence, cancer specific mortality and all-cause mortality. These findings remained significant after adjusting for clinicopathological features, indicating an independent association of high pretreatment PLR with adverse outcomes.

An exact explanation for this observation remains unclear and is yet to be elucidated. The PLR represents a marker of inflammation. A high PLR reflects both an elevated platelet dependent pro-tumor reaction and a decreased lymphocyte mediated anti-tumor immune response, which may both contribute to cancer progression and poor outcome. Platelets have been shown to promote cancer growth and metastasis. For instance, 
Table 3 Univariate analyses of various clinical parameters in prostate cancer patients

\begin{tabular}{|c|c|c|c|c|c|c|}
\hline \multirow[t]{2}{*}{ Parameters } & \multicolumn{2}{|c|}{ Progression-Free survival } & \multicolumn{2}{|l|}{ Cancer-Specific survival } & \multicolumn{2}{|l|}{ Overall survival } \\
\hline & $\mathrm{HR}(95 \% \mathrm{Cl})$ & $P$-value & $\mathrm{HR}(95 \% \mathrm{Cl})$ & $P$-value & $\mathrm{HR}(95 \% \mathrm{Cl})$ & $P$-value \\
\hline Age (years) & $0.958(0.938-0.981)$ & $<0.001$ & $0.993(0.962-1.024)$ & 0.641 & $1.005(0.975-1.035)$ & 0.749 \\
\hline PSA ( $\mu \mathrm{g} / \mathrm{L})$ & $1.003(1.002-1.004)$ & $<0.001$ & $1.003(1.001-1.005)$ & 0.002 & $1.002(1.000-1.004)$ & 0.029 \\
\hline Gleason Score & & $<0.001$ & & $<0.001$ & & $<0.001$ \\
\hline$\leq 7$ & 1 & & 1 & & 1 & \\
\hline$>7$ & $2.791(1.921-4.055)$ & & $4.710(2.499-8.878)$ & & 4.998 (2.286-6.993) & \\
\hline Metastasis & & $<0.001$ & & $<0.001$ & & $<0.001$ \\
\hline No & 1 & & 1 & & 1 & \\
\hline Yes & 3.447 (2.385-4.984) & & $5.893(3.128-11.102)$ & & $3.951(2.331-6.696)$ & \\
\hline Risk Stratification & & 0.005 & & 0.058 & & 0.111 \\
\hline Lowintermediate & 1 & & 1 & & 1 & \\
\hline High & $4.128(1.524-11.182)$ & & $6.751(0.935-48.730)$ & & $2.560(0.805-8.140)$ & \\
\hline Platelet $\left(10^{9} / \mathrm{L}\right)$ & & 0.053 & & 0.157 & & 0.121 \\
\hline$<190.50$ & 1 & & 1 & & 1 & \\
\hline$\geq 190.50$ & $1.414(0.995-2.003)$ & & $1.442(0.869-2.394)$ & & $1.450(0.907-2.318)$ & \\
\hline Lymphocyte $\left(10^{9} / \mathrm{L}\right)$ & $0.866(0.632-1.185)$ & 0.367 & $0.724(0.454-1.153)$ & 0.174 & $0.775(0.506-1.188)$ & 0.242 \\
\hline PLR & & 0.029 & & 0.014 & & 0.020 \\
\hline$<117.58$ & 1 & & 1 & & 1 & \\
\hline$\geq 117.58$ & $1.480(1.040-2.107)$ & & $1.948(1.145-3.312)$ & & $1.781(1.096-2.894)$ & \\
\hline
\end{tabular}

Abbreviations: $H R$ hazard ratio, $\mathrm{Cl}$ confidence interval, $P S A$ prostate-specific antigen

Boucharaba A et al. [21] showed platelet-derived lysophosphatidic acid was critical for bone metastasis formation in breast cancer. Dashevsky $\mathrm{O}$ et al. [22] demonstrated platelet-derived microparticles promoted invasiveness of $\mathrm{PCa}$ cells via upregulation of MMP-2 production. Zheng $\mathrm{S}$ et al. [23] found, in $\mathrm{PCa}$, fibrinogen help platelets to adhere to tumor cells, and platelets in turn promoted more fibrinogen to aggregate around tumor cells by forming thrombin, and thus protected tumor cells from natural killer cell cytotoxicity, which was mediated by $\beta 3$-integrins. There is considerable evidence that lymphocytes represent the cellular basis of cancer immunosurveillance, which inhibit tumor cell proliferation and metastasization [24]. Huang $\mathrm{SH}$ et al. [25] showed pretreatment high circulating lymphocytes could predict better recurrence-free survival and marginally better overall survival in $\mathrm{HPV}^{+}$oropharyngeal cancer patients. Adams S et al. [26] confirmed tumor stromal lymphocytic infiltration constituted a robust prognostic factor in triple-negative breast cancers from

Table 4 Multivariate analyses of various clinical parameters in prostate cancer patients

\begin{tabular}{|c|c|c|c|c|c|c|}
\hline \multirow[t]{2}{*}{ Parameters } & \multicolumn{2}{|c|}{ Progression-Free survival } & \multicolumn{2}{|c|}{ Cancer-Specific survival } & \multicolumn{2}{|l|}{ Overall survival } \\
\hline & $\mathrm{HR}(95 \% \mathrm{Cl})$ & $P$-value & HR $(95 \%$ Cl) & $P$-value & $\mathrm{HR}(95 \% \mathrm{Cl})$ & $P$-value \\
\hline Age (years) & $0.975(0.955-0.996)$ & 0.023 & & - & & - \\
\hline PSA $(\mu \mathrm{g} / \mathrm{L})$ & & 0.093 & & 0.916 & & 0.480 \\
\hline Gleason Score & & $<0.001$ & & $<0.001$ & & $<0.001$ \\
\hline$\leq 7$ & 1 & & 1 & & 1 & \\
\hline$>7$ & $2.136(1.454-3.137)$ & & $3.378(1.776-6.428)$ & & $3.086(1.747-5.453)$ & \\
\hline Metastasis & & $<0.001$ & & $<0.001$ & & $<0.001$ \\
\hline No & 1 & & 1 & & 1 & \\
\hline Yes & $2.830(1.939-4.131)$ & & $4.505(2.367-8.573)$ & & $3.080(1.799-5.274)$ & \\
\hline PLR & & 0.013 & & 0.037 & & 0.044 \\
\hline$<117.58$ & 1 & & 1 & & 1 & \\
\hline$\geq 117.58$ & $1.581(1.100-2.272)$ & & $1.768(1.036-3.015)$ & & $1.650(1.013-2.687)$ & \\
\hline
\end{tabular}


two phase III randomized adjuvant breast cancer trials. However, the differences of tumor features including age, serum PSA level, Gleason score, risk stratification and incidence of metastasis between low PLR group $(<117.58)$ and high PLR group $(\geq 117.58)$ were not significant in our study.

To date, adverse clinical outcomes of many cancers have been associated with an elevated PLR [8-15]. Zhou X et al. [27] performed a meta-analysis on the prognostic value of PLR in various cancers, and found that elevated PLR was a negative predictor for OS with HR of 1.60 (95 \% CI 1.351.90, $P<0.001)$. Similarly, Templeton AJ et al. [28] conducted a systematic review and showed that a high PLR was associated with worse OS in various solid tumors.

In contrast to the well-known prognostic value of PLR in other malignancies, its value in PCa is poorly studied. Only two studies exist regarding the association of PLR and PCa outcomes. In 384 patients treated with 3D conformal radiotherapy from 1999 to 2007, Langsenlehner T et al. [18] noted an increased PLR $(\geq 190)$ was a significant prognostic factor for poor distant metastases-free survival, cancer-specific survival and overall survival. Importantly, intergroup inconsistent neo-adjuvant therapy and potential confounding factors like inflammatory diseases may affect their study results. In 2015, Li F et al. [29] evaluated the relationship between PLR $\geq 150$ and all cause mortality in 103 PCa patients, and found that PLR was an independent risk factor of 3-year mortality, However, their analysis was limited by its relatively small patient population and intergroup inconsistent therapy.

To the best of our knowledge, our analysis is the first study of PLR on the prognosis of PCa treated with ADT, and strikingly our results showed that PLR was an independent prognostic factor for PFS, CSS and OS. Comparing to previous studies, we made a subgroup analysis and built a new integrated prognostic model. In subgroup analysis, we found that high PLR could predict poor prognosis in the subgroup of patients with Gleason score $>7$ or bone metastasis. In the subgroup of Gleason score $\leq 7$ or non-metastasis, however, PLR was not statistically significantly associated with prognosis, probably because the percentages of patients who reached the endpoints (progression, cancer-related death and overall death) in this subgroup were too small. With the traditional prognostic base model, which includes only Gleason score and incidence of metastasis, the predictive accuracy was 73.0, 77.8 and $74.6 \%$ for PFS, CSS and OS, respectively. The predictive accuracy was clearly improved by the addition of PLR (74.7, 80.1 and $76.8 \%$ ).

There are some limitations to our current study. First, this was a retrospective investigation. Despite the strict enrollment criteria applied, we were unable to completely exclude conditions that might cause inflammatory changes in PCa. Second, the patient data were collected from a single institution. Our results need to be validated by prospective research and patient data from multiple medical centers.

\section{Conclusion}

PLR might be a novel prognostic marker in predicting the clinical outcome for PCa patients treated with ADT. Thus, we recommend adding PLR to traditional prognostic model to improve the predictive accuracy.

\section{Additional file}

Additional file 1: Original data of 290 prostate cancer patients receiving androgen deprivation therapy. (XLS $70 \mathrm{~kb}$ )

\section{Abbreviations}

ADT: androgen deprivation therapy; Cl: confidence interval; CSS: cancerspecific survival; HR: hazard ratio; IQR: interquartile range; NLR: neutrophil to lymphocyte ratio; OS: overall survival; PCa: prostate cancer; PFS: progressionfree survival; PLR: Platelet to Lymphocyte ratio; PSA: prostate-specific antigen.

\section{Acknowledgements}

We sincerely thank the patients for their participation in this study.

\section{Funding}

This study was supported by National Natural Science Foundation of China (91129725, 81572536), Science and Technology Commission of Shanghai Municipality (14140901700), Shanghai Municipal Education Commission (15ZZ058), Key Disciplines Group Construction Project of Pudong Health Bureau of Shanghai (PWZxq2014-05), the Joint research foundation of Shanghai Shenkang hospital development center on innovative medical technology (SHDC12015125), Shanghai Municipal Education CommissionGaofeng Clinical Medicine Grant Support (20152215), and the Shanghai Jiao Tong University School of Medicine Translational Research Innovation Fund (15ZH4002). The funders had no role in study design, data collection and analysis, decision to publish, or preparation of the manuscript.

\section{Availability of data and materials}

You can see the data and materials in Additional file 1 named "original data.xls".

\section{Authors' contributions}

WYQ, DBJ and XW were responsible for the design of the study. WYQ, XF and HYR contributed to the manuscript composition, submission and revision. PJH, ZYJ, SXG, SJJ and LQ participated in the data collection, interpretation and discussion. WZZ and CY participated in the data analysis and manuscript mapping. DBJ, XW and HYR was responsible for the funding application and the supervision and management of the project. All authors have contributed to and approved the final manuscript.

\section{Competing interest}

The authors declare that they have no competing interests.

\section{Consent to publish}

We had obtained the consents to publish from the participants to report individual patients' data in any form (including images, videos, voice recordings etc.).

\section{Ethics, consent and permissions}

The study was approved by the Ethical Committee of Renji Hospital, Shanghai Jiao Tong University School of Medicine. Consents to participate in the study from the participants were obtained.

\section{Author details}

'Department of Urology, Renji Hospital, School of Medicine, Shanghai Jiao Tong University, 1630 Dongfang Road, Pudong District, Shanghai 200127, People's Republic of China. ${ }^{2}$ School of Public Health, Shanghai Jiao Tong University, Shanghai, China. ${ }^{3}$ Department of Pathology, Renji Hospital, School of Medicine, Shanghai Jiao Tong University, Shanghai, China. 
Received: 28 December 2015 Accepted: 16 May 2016 Published online: 24 May 2016

\section{References}

1. Siegel R, Miller KD, Jemal A. Cancer statistics, 2015. CA Cancer J Clin. 2015; 65(1):5-29.

2. Wein AJ, Kavaussi LR, Novick AC, Partin AW, Peters CA, Nelson JB, et al. Campbell-Wash urology. 10th ed. Philadelphia: Elsevier; 2012. p. 2934-53.

3. De Nunzio C, Kramer G, Marberger M, Montironi R, Nelson W, Schröder F, et al. The controversial relationship between benign prostatic hyperplasia and prostate cancer: the role of inflammation. Eur Urol. 2011;60(1):106-17.

4. Sfanos KS, De Marzo AM. Prostate cancer and inflammation: the evidence. Histopathology. 2012;60(1):199-215.

5. Taverna G, Pedretti E, Di Caro G, Borroni EM, Marchesi F, Grizzi F. Inflammation and prostate cancer: friends or foe? Inflamm Res. 2015;64(5):275-86.

6. Fujita K, Imamura R, Tanigawa G, Nakagawa M, Hayashi T, Kishimoto N, et al. Low serum neutrophil count predicts a positive prostate biopsy. Prostate Cancer Prostatic Dis. 2012;15(4):386-90.

7. Langsenlehner T, Thurner EM, Krenn-Pilko S, Langsenlehner U, Stojakovic T, Gerger A, et al. Validation of the neutrophil-to lymphocyte ratio as a prognostic factor in a cohort of European prostate cancer patients. World J Urol. 2015 Jan 24. [Epub ahead of print]

8. Wang DS, Ren C, Qiu MZ, Luo HY, Wang ZQ, Zhang DS, et al. Comparison of the prognostic value of various preoperative inflammation-based factors in patients with stage III gastric cancer. Tumour Biol. 2012;33(3):749-56.

9. Stotz M, Gerger A, Eisner F, Szkandera J, Loibner H, Ress AL, et al. Increased neutrophil-lymphocyte ratio is a poor prognostic factor in patients with primary operable and inoperable pancreatic cancer. Br J Cancer. 2013;109(2):416-21.

10. Asher V, Lee J, Innamaa A, Bali A. Preoperative platelet lymphocyte ratio as an independent prognostic marker in ovarian cancer. Clin Transl Oncol. 2011;13(7):499-503.

11. Kwon HC, Kim SH, Oh SY, Lee S, Lee JH, Choi HJ, et al. Clinical significance of preoperative neutrophil-lymphocyte versus platelet-lymphocyte ratio in patients with operable colorectal cancer. Biomarkers. 2012;17(3):216-22.

12. Liu H, Wu Y, Wang Z, Yao Y, Chen F, Zhang H, et al. Pretreatment plateletto-lymphocyte ratio (PLR) as a predictor of response to first-line platinumbased chemotherapy and prognosis for patients with non-small cell lung cancer. J Thorac Dis. 2013;5(6):783-9.

13. Pinato DJ, Stebbing J, Ishizuka M, Khan SA, Wasan HS, North BV, et al. A novel and validated prognostic index in hepatocellular carcinoma: the inflammation based index (IBI). J Hepatol. 2012;57(5):1013-20.

14. Fox P, Hudson M, Brown C, Lord S, Gebski V, De Souza P, et al. Markers of systemic inflammation predict survival in patients with advanced renal cell cancer. Br J Cancer. 2013;109(1):147-53.

15. Dutta S, Crumley AB, Fullarton GM, Horgan PG, McMillan DC. Comparison of the prognostic value of tumour- and patient-related factors in patients undergoing potentially curative resection of esophageal cancer. World J Surg. 2011;35(8):1861-6.

16. Kaynar M, Yildirim ME, Gul M, Kilic O, Ceylan K, Goktas S. Benign prostatic hyperplasia and prostate cancer differentiation via platelet to lymphocyte ratio. Cancer Biomark. 2015;15(3):317-23.

17. Yuksel $\mathrm{OH}$, Urkmez A, Akan S, Yldirim C, Verit A. Predictive value of the platelet-to-lymphocyte ratio in diagnosis of prostate cancer. Asian Pac J Cancer Prev. 2015;16(15):6407-12.

18. Langsenlehner T, Pichler M, Thurner EM, Krenn-Pilko S, Stojakovic T, Gerger A, et al. Evaluation of the platelet-to-lymphocyte ratio as a prognostic indicator in a European cohort of patients with prostate cancer treated with radiotherapy. Urol Oncol. 2015;33(5):201.e9-16.

19. Heidenreich A, Bastian PJ, Bellmunt J, Bolla M, Joniau S, van der Kwast T, et al. EAU guidelines on prostate cancer. part 1: screening, diagnosis, and local treatment with curative intent-update 2013. Eur Urol. 2014;65(1):124-37.

20. Heidenreich A, Bastian PJ, Bellmunt J, Bolla M, Joniau S, van der Kwast T, et al. EAU guidelines on prostate cancer. part II: Treatment of advanced, relapsing, and castration-resistant prostatecancer. Eur Urol. 2014;65(2):467-79.

21. Boucharaba A, Serre CM, Gres S, Saulnier-Blache JS, Bordet JC, Guglielmi J, et al. Platelet-derived lysophosphatidic acid supports the progression of osteolytic bone metastases in breast cancer. J Clin Invest. 2004;114(12):1714-25.

22. Dashevsky O, Varon D, Brill A. Platelet-derived microparticles promote invasiveness of prostate cancer cells via upregulation of MMP-2 production. Int J Cancer. 2009;124(8):1773-7.
23. Zheng S, Shen J, Jiao Y, Liu Y, Zhang C, Wei M, et al. Platelets and fibeinogen facilitate each other in protecting tumor cells from natural killer cytotoxicity. Cancer Sci. 2009;100(5):859-65.

24. Dunn GP, Old LJ, Schreiber RD. The immunobiology of cancer immunosurveillance and immunoediting. Immunity. 2004;21(2):137-48.

25. Huang SH, Waldron JN, Milosevic M, Shen X, Ringash J, Su J, et al. Prognostic value of pretreatment circulating neutrophils, monocytes, and lymphocytes in oropharyngeal cancer stratified by human papillomavirus status. Cancer. 2015; 121(4):545-55.

26. Adams S, Gray RJ, Demaria S, Goldstein L, Perez EA, Shulman LN, et al. Prognostic value of tumor-infiltrating lymphocytes in triple-negative breast cancers from two phase III randomized adjuvant breast cancer trials: ECOG 2197 and ECOG 1199. J Clin Oncol. 2014;32(27):2959-66.

27. Zhou X, Du Y, Huang Z, Xu J, Qiu T, Wang J, et al. Prognostic value of PLR in various cancers: a meta-analysis. PLoS One. 2014;9(6), e101119.

28. Templeton AJ, Ace O, McNamara MG, Al-Mubarak M, Vera-Badillo FE, Hermanns $T$, et al. Prognostic role of platelet to lymphocyte ratio in solid tumors: a systematic review and meta-analysis. Cancer Epidemiol Biomarkers Prev. 2014; 23(7):1204-12

29. Li F, Hu HB, Gu S, Chen X, Sun Q. Platelet to lymphocyte ratio plays an important role in prostate cancer's diagnosis and prognosis. Int J Clin Exp Med. 2015:8(7):11746-51.

\section{Submit your next manuscript to BioMed Central and we will help you at every step:}

- We accept pre-submission inquiries

- Our selector tool helps you to find the most relevant journal

- We provide round the clock customer support

- Convenient online submission

- Thorough peer review

- Inclusion in PubMed and all major indexing services

- Maximum visibility for your research

Submit your manuscript at www.biomedcentral.com/submit
) Biomed Central 